\title{
artículos
}

\section{El prontuario como indicador del uso de los recursos informativos de la universidad}

\section{Fabio Restrepo L}

Universidad Interamericana de Puerto Rico, Recinto de San Germán, Call Box 5100, San Germán, Puerto Rico

\begin{abstract}
RESUMEN

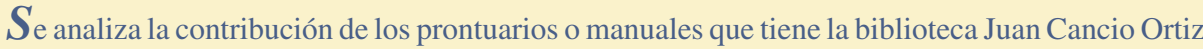
de la Universidad Interamericana de Puerto Rico, sobre la utilización de los recursos informativos y de investigación. Las hipótesis iniciales de trabajo de que tales prontuarios no estimulan significativamente el uso de los recursos de la biblioteca, y de que existe poca participación de la facultad en el desarrollo de los recursos de la biblioteca, se confirmaron afirmativamente: los prontuarios no estimulan y existe poca participación..
\end{abstract}

\begin{abstract}
$\boldsymbol{T}$ he contribution of use manuals in relation to the information and research resources in the Juan Cancio Ortiz Library of the Universidad Interamericana de Puerto Rico, is here analyzed. The initial work hypothesis that such manuals do not significantly stimulate the use of library resources is confirmed in the conclusions. The suspicion that the faculty of the university do not participate in the development of library resources, is also confirmed by results.
\end{abstract}

\section{INTRODUCCIÓN}

$\boldsymbol{L}^{\mathrm{a}}$ biblioteca, como el centro de recursos para el aprendizaje más importante de la universidad, no siempre ha respondido a las expectativas de los administradores académicos, de los profesores, de los alumnos y de los bibliotecarios. Con frecuencia los recursos informativos disponibles en la biblioteca no se utilizan en todo su potencial.

Las causas de esta subutilización abarcan un amplio espectro que va desde el poco estímulo que se recibe en el aula hasta el ambiente poco propicio para el estudio que ofrece la sala de lectura, así como la falta de orientación adecuada sobre cómo usar las fuentes de información disponibles en la biblioteca.
Desde luego, las bibliotecas universitarias no son las únicas subutilizadas, pero si se compara el costo global de establecer y mantener un adecuado nivel de servicio para una comunidad académica, el problema adquiere otra dimensión. En la actualidad, los precios de los materiales impresos y no impresos que deben adquirir las bibliotecas de universidades son muy altos y la tendencia es hacia el alza cada vez mayor.

Entre las soluciones propuestas para aliviar la situación, se destacan las siguientes: programas de adiestramiento en el uso de los recursos para profesores, alumnos y administradores, un mayor acercamiento entre los bibliotecarios y los usuarios de sus servicios, reducción en los niveles de adquisición de recursos y los programas de cooperación interbibliotecaria.

El propósito principal de esta investigación fue analizar la contribución que los prontuarios de los cursos que se ofrecen en la Universidad Interamericana, Recinto de San Germán, hacen a la utilización de los recursos informativos e investigativos, impresos y no impresos que existen y se obtienen constantemente en la Biblioteca Juan Cancio Ortiz.

Como en muchas de las investigaciones que con frecuencia se realizan, la respuesta de las personas a las cuales se les solicitó información pertinente no fue muy satisfactoria, lamentablemente. Sin embargo, los datos obtenidos se consideraron repre- 


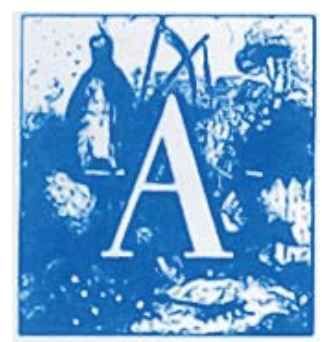

Tanto los materiales existentes en la biblioteca, como los que continuamente se adquieren para mantener actualizada su colección, deben utilizarse al máximo para poder justificar la inversión.

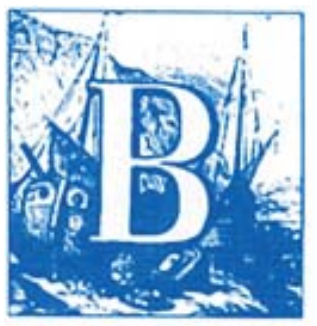

sentativos de la situación existente en la universidad y sirvieron para cumplir con los propósitos de la investigación.

\section{PREGUNTA DE INVESTIGACION}

¿Estimulan los prontuarios de los cursos del Recinto de San Germán el uso de los diferentes recursos informativos disponibles en, y por intermedio de, la biblioteca?

La función de intermediario de la biblioteca se refiere a las múltiples alternativas que ésta le presenta a

los usuarios cuando la información o el material solicitado no se encuentran disponibles, directa e inmediatamente. Tal es el caso por ejemplo, de préstamos interbibliotecarios y la consulta de bases de datos a través de DIALOG.

\section{IMPORTANCIA DEL ESTUDIO}

Tanto los materiales existentes en la biblioteca, como los que continuamente se adquieren para mantener actualizada su colección, deben utilizarse al máximo para poder justificar la inversión. De no ser así, hay desperdicio de recursos y el proceso enseñanza-aprendizaje resulta ser, en primer instancia, el más afectado. La investigación buscó establecer, objetivamente, la participación de los profesores de la universidad en la promoción del uso de los recursos de la biblioteca, por medio de los prontuarios de sus clases.

Indirectamente, el proyecto estuvo encaminado a detectar el flujo de comunicación entre la facultad y los bibliotecarios, en cuanto a los recursos de información que exigen los cursos, para poder así sugerir cambios que contribuyan a fortalecer la interacción entre unos y otros. Es posible que una inadecuada comunicación afecte negativamente la función de promoción del uso de los recursos que corresponde a la facultad, y que la no participación de los bibliotecarios en diferentes actividades relacionadas con el currículo y la instrucción afecte el desarrollo de los recursos de la biblioteca adversamente.

\section{OBJETIVOS}

Los objetivos del proyecto fueron los siguientes:

1. Determinar el nivel al cual los prontuarios de los cursos contribuyen a la utilización de los recursos de información disponibles en la biblioteca.
2. Analizar las metodologías mayormente utilizadas por los profesores de la universidad para impartir la instrucción y categorizar la contribución que cada una de ellas hace al uso de los recursos de la biblioteca.

3. Revisar la fecha de publicación de las referencias bibliográficas incluidas en los prontuarios y confrontar aleatoriamente la existencia de materiales similares más recientes en la biblioteca o en fuentes de información pertinentes.

4. Verificar aleatoriamente la disponibilidad en la biblioteca de los materiales recomendados por los profesores para sus cursos.

5. Determinar el grado de participación de los profesores en el proceso de selección de materiales para la biblioteca.

6. Recomendar estrategias para aumentar la utilización de los recursos de la biblioteca.

\section{SUPUESTOS Y LIMITACIONES}

Esta investigación partió de dos supuestos:

1. Que los prontuarios no estimulan significativamente el uso de los recursos de la biblioteca.

2. Que existe poca participación de la facultad en el desarrollo de los recursos de la biblioteca.

Una limitación importante en este estudio fue la dificultad de asignar un valor indicativo de mayor o menor estímulo al uso de los recursos, a las diferentes modalidades de instrucción de la facultad. Sin embargo, mediante el uso de categorías empleadas en estudios semejantes, se redujo en gran medida el factor de arbitrariedad que podría estar presente.

Una variable sobre la cual el estudio fue la dificultad de asignar un valor indicativo de mayor o menor estímulo al uso de los recursos, a las diferentes modalidades de instrucción de la facultad. Sin embargo, mediante el uso de categorías empleadas en los estudios semejantes, se redujo en gran medida el factor de arbitrariedad que podría estar presente. Una variable sobre la cual el estudio no intentó ejercer un estricto control, fue el estímulo oral que, formal o informalmente, muchos miembros de la facultad le dan a sus estudiantes para que usen la biblioteca, aunque no aparezca evidencia de ello en los prontuarios. 


\section{ALCANCE}

La investigación estuvo dirigida al análisis del estímulo que los prontuarios de los cursos le dan al uso de los recursos de la biblioteca y no pretendió, de ninguna manera, evaluar la metodología que cada profesor selecciona para su curso. Asimismo, buscó detectar la presencia de factores que inciden en la efectiva comunicación entre los bibliotecarios y la facultad en relación con los cursos y los materiales de información e investigación que requieren dichos cursos.

El análisis aleatorio de la fecha de publicación de los materiales bibliográficos contenidos en los prontuarios, tuvo como único objeto estimular el diálogo entre bibliotecarios y profesores acerca de la posibilidad de actualizar algunas de las referencias.

\section{DEFINICIONES}

Para este estudio, el uso de la biblioteca se ha definido como la acción coordinada, sistemática y bajo control individual, de aprovechar las diferentes herramientas bibliográficas y no bibliográficas (catálogo en línea, libros de referencia, libros de la colección general, índices, revistas, etc.) existentes en la biblioteca, que facilitan el acceso a la información necesaria para iniciar o completar una asignación, monografía o trabajo de investigación.

De igual manera, para los propósitos de la investigación, el estímulo de los prontuarios al uso de los recursos bibliotecarios, se refiere primordialmente a la dinámica del uso de la biblioteca, como se definió en el párrafo anterior, que genera en los estudiantes las diferentes estrategias empleadas por la facultad en el desarrollo de sus clases.

\section{REVISION DE LA LITERATURA}

Linda Rambler (1982) en un estudio de los prontuarios de una universidad, observó que los modos de instrucción, en conjunto, tienden a hacer poco uso de los recursos bibliotecarios. Expresó, además, que:

[...] el conocimiento que se obtiene de las prácticas académicas con el estudio de los prontuarios, sirve para facilitar acciones bibliotecarias orientadas hacia un programa de integración curricular y crea componentes adicionales para tomar decisiones en áreas como la distribución presupuestal, el desarrollo de colecciones y la asignación de personal bibliotecario.

Posteriormente, Lauer, Merz y Craig (1985), después de analizar los prontuarios de dos instituciones académicas privadas -una con orientación a las artes liberales y la otra con programas en artes liberales y educación profesional-, concluyeron que su estudio presenta evidencia adicional de que el uso de la biblioteca por parte de los estudiantes subgraduados es bajo. Concluyeron, además, que "al menos en las dos instituciones estudiadas, el énfasis curricular es un mal previsible del uso relativo de la biblioteca, aunque el currículo orientado a las profesiones evidenció un nivel un poco más alto de uso requerido".

Sayles (1985) en una investigación sobre las posibles aplicaciones del análisis de los prontuarios dice que El prontuario, una expansión lógica de la descripción de un curso en forma de bosquejo de estudio para el estudiante, es una mina de oro de información de la cual se puede extraer material para una guía de estudio y otras aplicaciones". Entre éstas señala como importantes las siguientes: clarificación de las asignaciones, desarrollo de la colección, preparación de guías informativas de la biblioteca (pathfinders), servicio de referencia anticipado, instrucción bibliográfica y mejoramiento de los cursos. En esta última aplicación, el autor cita a Robert Spencer (1978) quien expresa que "[...la calidad de la biblioteca está relacionada con la calidad de la enseñanza]". Y Agrega: "[...si los profesores mismos desconocen o no acostumbran diseñar clases y experiencias de aprendizaje alrededor de la biblioteca, sus estudiantes van a responder de la misma manera]".

En un estudio relacionado, Barbara Golden (1984) habla de la evaluación de la colección de una biblioteca tomando como base el examen de los cursos ofrecidos por la universidad según aparecen descritos en el catálogo y en los prontuarios. Un procedimiento similar utilizó John W. Whaley (1981) para el análisis de una colección, aunque él prefirió asignar números de clasificación a cada una de las partes de los prontuarios, a partir de los términos "libres" que los profesores aportaron cuando así se les solicitó.
En resumen, los estudios sobre el análisis de los prontuarios para determinar la interacción entre curriculo-profesores y recursos-bibliotecarios apoyaron y sirvieron de base para la investigación realizada.

\section{METODOLOGIA}

Para realizar este proyecto se procedió de la siguiente manera:

1. Se seleccionó una muestra aleatoria (lista de números aleatorios) de cursos ofrecidos en el Recinto, y se tomó como base el programa de clases del segundo semestre 1991-92.

2. Se solicitaron y obtuvieron en la secretaría de los diferentes departamentos los prontuarios respectivos.

3. Se procedió al análisis de las metodologías usadas por los profesores en el proceso de instrucción. A cada tipo de método, por ejemplo, conferencia, presentaciones audiovisuales, monografía, se le asignó un valor de "mucho", "algo" o "nada" de acuerdo con el efecto motivador que dicho método puede tener sobre el uso de la biblioteca. Una monografía se cnsideró que contribuye "mucho" al uso, y una conferencia, "nada". La diferencia entre las tres categorías está en la evaluación de cuánta búsqueda y cuánto uso de los recursos de la biblioteca debe realizar un estudiante para encontrar información relevante para satisfacer los requisitos del curso. Estas categorías corresponden a las utilizadas por Linda Rambler (1982) en su investigación de prontuarios y se adoptaron por considerarlas adecuadas para el presente estudio.

4. Aleatoriamente se seleccionó un grupo representativo de referencias bibliográficas contenidas en los prontuarios y se verificó:

a) Su presencia en la biblioteca.

b) Si la fecha de publicación correspondía a la edición más recientemente publicada de la obra, en el caso de monografías. En el caso de artículos de revistas, no se intentó determinar la existencia de artículos similares más recientes, no sólo por la dificultad de encontrarlos sino porque es bastante probable que en la literatura reciente existan materiales más actuales. 
5. Se preparó un breve cuestionario para los profesores de los cursos seleccionados en la muestra, a fin de obtener información sobre otros métodos de motivar el uso de los recursos de la biblioteca y su participación en la selección de esos recursos.

\section{RESULTADOS DE LA INVESTIGACION}

De un total de 1.015 cursos ofrecidos en el Recinto en el Segundo semestre 1991-92, según el programa de clases, se seleccionó una muestra de 278, de acuerdo con la tabla utilizada por Krejcie y Morgan (1970) para determinar el tamaño de una muestra. La selección de los 278 cursos de los cuales se conseguirían prontuarios, se hizo con una tabla de 10,000 dígitos aleatorios.

De los 278 prontuarios solicitados en las secretarías de los programas correspondientes a los prontuarios seleccionados, solamente se recibieron 140 , o sea un poco más del $50 \%$, de los cuales 3 no pudieron usarse por estar incompletos y cuatro por estar duplicados. Varios factores pudieron haber contribuido a la baja respuesta, entre ellos la cancelación que normalmente ocurre de algunos cursos, la inexistencia de prontuarios en otros casos, la entrega de sólo un prontuario cuando resultaron seleccionadas varias secciones del mismo curso, el traslado del profesor a otro recinto o trabajo $\mathrm{y}$, por supuesto, la tadicional indiferencia hacia solicitudes de información por parte de investigadores. El investigador considera, sin embargo, que la muestra es adecuada para los propósitos del presente estudio.

\begin{tabular}{|c|c|c|}
\hline \multicolumn{3}{|c|}{$\begin{array}{c}\text { Tabla } 1 \\
\text { Modos más frecuentes organizados en } \\
\text { orden descendente }\end{array}$} \\
\hline & $\mathrm{f}^{*}$ & $\%$ \\
\hline Texto & 84 & 63 \\
\hline Lecturas & 45 & 34 \\
\hline Laboratorios & 37 & 27 \\
\hline Ejercicios & 34 & 26 \\
\hline Presentaciones orales & 33 & 25 \\
\hline Trabajos escritos & 25 & 19 \\
\hline Proyectos & 25 & 19 \\
\hline \multicolumn{3}{|l|}{$\mathrm{f}^{*}=$ en la muestra } \\
\hline
\end{tabular}

El análisis de los 133 prontuarios, que se presenta más adelante (tabla 3 ) acompañado de número y porcentajes, indica que, el $55 \%$ de los cursos, aproximadamente, no exige el uso de la biblioteca, $31 \%$ exige algo si solamente el $6 \%$ exige mucho uso. El cálculo de la aproximación de la distribución normal al binomio establece la fluctuación de posibles valores del porcentaje real alrededor del porcentaje $P$ de la muestra. El 95\% de probabilidad $(P-3 x$ a $P+3 x)$ produce la fluctuación de la población. La estadística indica que no hay uso de la biblioteca entre, aproximadamente, el $44 \%$ y el $66 \%$ del total de cursos que se ofrecen en la institución. Se usa un poco la biblioteca entre el $22 \%$ y el $39 \%$ de los cursos y mucho entre el $4 \%$ y el $8 \%$ de ellos.

\section{ANALISIS DE METODOLOGIAS CONSIGNADAS EN LOS PRONTUARIOS}

La tabla 2 muestra el análisis de las diferentes metodologías consignadas por los profesores en los prontuarios sometidos a estudio y de su análisis se desprenden varias observaciones importantes:

El número de cursos que no usan prontuario no fue posible determinarlo porque tampoco pudo saberse la causa por la cual no se dio respuesta a la solicitud de envío.

Exceptuando los exámenes y los quizzes (encuestas), dos categorías que no podrían considerarse como métodos de enseñanza, la modalidad de instrucción que más predomina entre los prontuarios examinados es el libro de texto con un total de

\begin{tabular}{|c|c|c|c|c|c|}
\hline Modos de instrucc & $\begin{array}{r}T a b \\
\text { mencionado }\end{array}$ & $\begin{array}{l}\text { la } 2 \\
\text { s en los pro }\end{array}$ & tuarios de & uestra. & \\
\hline Metodología & Número de & $\%$ de uso & Nivel de es & lo al usc & biblioteca \\
\hline & usos & & Mucho & Algo & $\mathrm{Nada}$ \\
\hline No usan prontuarios & $?$ & & & & \\
\hline Pront. sin metodología & 11 & 8 & & & \\
\hline Asignaciones en revistas & 7 & 5 & $\mathrm{X}$ & & \\
\hline Conferencias & 14 & 11 & & & $\mathrm{X}$ \\
\hline Demostración & 3 & 2 & & & $\mathrm{X}$ \\
\hline Discusión en clase & 21 & 16 & & & $\mathrm{X}$ \\
\hline Ejercicios asignados & 34 & 26 & & & $\mathrm{X}$ \\
\hline Estudios individuales & 2 & 1.5 & & $\mathrm{X}$ & \\
\hline Estudios de casos & 11 & 8 & & $\mathrm{X}$ & \\
\hline Exámenes & 117 & 88 & & & $\mathrm{X}$ \\
\hline Investigaciones & 3 & 2 & & $\mathrm{X}$ & \\
\hline Laboratorios & 37 & 28 & & & $\mathrm{X}$ \\
\hline Lecturas (mimeo hand.) & 5 & 4 & & & $\mathrm{X}$ \\
\hline Lecturas asignadas & 36 & 27 & & $X$ & \\
\hline Lecturas (No reservadas) & 4 & 3 & & $\mathrm{X}$ & \\
\hline Panel/Debate & 4 & 3 & & $\mathrm{X}$ & \\
\hline Participación & 20 & 15 & & & $\mathrm{X}$ \\
\hline Películas & 15 & 11 & & & $\mathrm{X}$ \\
\hline Práctica & 9 & 7 & & & $\mathrm{X}$ \\
\hline Presentaciones orales & 33 & 25 & & $\mathrm{X}$ & \\
\hline Proyectos (arte, juegos, diseños) & 25 & 19 & & & $\mathrm{X}$ \\
\hline Quizzes & 32 & 24 & & & $\mathrm{X}$ \\
\hline Revisión en Biblioteca & 3 & 2 & $\mathrm{X}$ & & $\mathrm{X}$ \\
\hline Seminarios & 1 & .7 & & & $\mathrm{X}$ \\
\hline Textos & 84 & 63 & & & $\mathrm{X}$ \\
\hline Trabajo de campo & 8 & 6 & & & $\mathrm{X}$ \\
\hline Trabajos escritos & 25 & 19 & & $\mathrm{X}$ & \\
\hline Visitantes a clase & 1 & .7 & & & $\mathrm{X}$ \\
\hline
\end{tabular}


84 usos o sea el $63 \%$ del total. Le siguen en frecuencia de uso las lecturas asignadas con un total de $34 \%$, una categoría que abarca tres diferentes modalidades: fotocopias que entrega el profesor a los estudiantes, lecturas de material en reserva en la biblioteca, tanto libros como fotocopias de otros tipos de material y lectura de material no disponible en reserva sino en otras secciones de la biblioteca, por ejemplo, la Colección Puertorriqueña, referencia o circulación. Los laboratorios ocupan el tercer lugar con un $27 \%$ y los ejercicios, el cuarto con $26 \%$.

De estas 4 categorías, sólo las lecturas asignadas que no se encuentran en la sección de reserva contribuyen algo al uso de la biblioteca. Las demás, desafortunadamente, se considera que no estimulan el uso de los recursos bibliotecarios, según se ha definido en este estudio. En el caso de lecturas asignadas que el estudiante encuentra en reserva en la biblioteca, el estudiante debe ir allí a buscar el material, pero no necesita usar siquiera el catálogo para obtenerlo, porque el sistema de reserva facilita la obtención del material mediante 1 nombre del profesor, únicamente.

Desde luego, para el estudiante resulta muy fácil obtener material de lectura de esa manera, pero al mismo tiempo se le priva de la oportunidad de investigar la existencia de otros materiales semejantes, quizás más relevantes, o más actualizados, en las diferentes fuentes de información existentes dentro y fuera de la biblioteca. A muchos profesores se les dificulta revisar la literatura que llega todos los días a la biblioteca y, muy a menudo, los estudiantes que por iniciativa propia consultan fuentes adicionales encuentran materiales relevantes desconocidos por el profesor.

Por otra parte, es importante recalcar que el libro de texto, por lo general, no contribuye en nada al uso de la biblioteca. Además, y como se verá más adelante, cuando se presentan los resultados de la verificación de los libros usados por los profesores para sus clases, la actualidad de la información que usualmente ofrece el libro de texto es cuestionable. Hoy en día la información en muchas áreas del conocimiento se actualiza tan constante y vertiginosamente que cuando un libro sale al mercado la información que contiene ya está desactualizada.
Los laboratorios y los ejercicios se considera que tampoco contribuyen al uso de la biblioteca. Los primeros por ser una actividad que se realiza directamente en el laboratorio, alejada de la biblioteca. Los segundos por tratarse de la práctica de destrezas o conocimientos adquiridos en clase. Es probable que ambas metodologías estimulen en alguna medida el uso de la biblioteca, pero para los efectos del presente estudio no se considera así.

Entre las categorías que generan "algún" uso de la biblioteca, se destacan en el estudio las presentaciones orales, con un $25 \%$. Sería interesante analizar el efecto de esta popular metodología en el aprovechamiento académico de los estudiantes.

Los trabajos escritos y los proyectos representan cada uno el $19 \%$ de frecuencia de uso como actividad educativa. La primera es una categoría que incluye diferentes modalidades, tales como ejercicios presentados por escrito, informes de visitas o de actividades de práctica, comentarios escritos de lecturas asignadas, resúmenes de artículos y otros, los cuales contribuyen sólo un poco al uso de la biblioteca. Los proyectos también presentan diferentes modalidades y pueden estimular el uso de la biblioteca de diferente manera.

Los estudios de casos contribuyen "algo" al uso de los recursos, pero no es una modalidad muy utilizada. Sólo se reportaron 11 veces en los prontuarios, para un $8 \%$ de representación en la muestra. Es una categoría que valdría la pena explorar adicionalmente.

Los estudios individuales sólo representan un $1 \%$ entre las modalidades, a pesar de su potencial de estímulo al uso de la biblioteca. Es otra categoría que quizás debería utilizarse con mayor frecuencia.

Las dos categorías que contribuyen mayormente al uso de la biblioteca son la búsqueda en biblioteca y las asignaciones en revistas, pero desafortunadamente ambas son modalidades de escasa utilización en el Recinto: el 2\% y el $5 \%$ respectivamente.

Es probable que otras modalidades de enseñanza contribuyan en igual medida que las dos anteriores al uso de la biblioteca. Sin embargo, como los prontuarios no mencionan específicamente esas categorías y en el presente estudio, como se explicó antes, las categorías se adoptaron de un estudio anterior similar, no es posi- ble, dentro del alcance de esta investigación, establecer esas contribuciones.

Las conferencias y los audiovisuales son dos categorías, con un $10 \%$ de representatividad cada una, que no contribuyen intrínsecamente al uso de la biblioteca. Empero, más adelante se presenta un resumen de los comentarios hechos por algunos profesores en respuesta a una pregunta realizada en una encuesta que se les hizo sobre otros estímulos no consignados en el prontuario. De ello se desprende que si bien es cierto que a veces en clase se presenta la ocasión para dirigir la atención del estudiante a ciertas fuentes de información en la biblioteca, o el profesor menciona un término o concepto que los estudiantes desconocen y que es importante que conozcan, también es cierto que se dificulta establecer el nivel de uso que generan tales hechos dadas las diversas maneras de presentar asignaciones que tienen los estudiantes. Algunos consultan directamente las fuentes para obtener la información deseada, mientras que otros se limitan a preguntarle al bibliotecario dónde encontrar la respuesta u obtienen de sus compañeros la información que necesitan para la asignación.

En relación con los audiovisuales, algunos profesores también indicaron, en la encuesta mencionada antes, que a partir de las presentaciones en la sala de audiovisuales de la biblioteca se estimula algún uso de los recursos, pero este hecho no es posible confirmarlo dentro del marco de la presente investigación.

Es importante recalcar un hecho significativo en las modalidades de enseñanza que, a juzgar por los prontuarios de la muestra, no se usan en el Recinto. Hay cuatro categorías, a saber: la instrucción asistida por computadora, la discusión, los invitados, y el panel/debate que no se mencionan. Valdría la pena indagar las razones por las cuales estas modalidades no se utilizan en el Recinto. Las demás categorías que aparecen en la Tabla 2 , en general, no estimulan el uso de la biblioteca.

En la Tabla 3 puede verse que los programas más representados en la muestra son igualmente los más numerosos en el Recinto. Varían sin embargo,, en ciertos aspectos significativos, por ejemplo: mientras que ciencias económicas y administrativas contribuyó al 24\% de los prontuarios, de ellos sólo el $1.5 \%$ estimu- 
Tabla 3

Resumen por Departamento o Programa

\begin{tabular}{|c|c|c|c|c|c|}
\hline Departamento o Programa & Cursos & $\%$ & Mucho (\%) & Algo (\%) & Nada $(\%)$ \\
\hline Sin metodología & $11 *$ & 8 & & & \\
\hline $\begin{array}{l}\text { Ciencias económicas y } \\
\text { administrativas }\end{array}$ & 32 & 24 & $2(1.5)$ & $11(8)$ & $19(14)$ \\
\hline $\begin{array}{l}\text { Biología y ciencias del } \\
\text { ambiente }\end{array}$ & 8 & 6 & $0(0)$ & $1(1)$ & $4(3)$ \\
\hline $\begin{array}{l}\text { Ciencias sociales y artes } \\
\text { liberales }\end{array}$ & 16 & 12 & $1(1)$ & $5(4)$ & $10(7)$ \\
\hline Educación & 26 & 19 & $3(2)$ & $12(9)$ & $11(8)$ \\
\hline Educación general (PEG) & 2 & 1.5 & $0(0)$ & & \\
\hline Enfermería & 1 & 1 & $0(0)$ & $1(1)$ & $0(0)$ \\
\hline Lenguas y literatura & 18 & 14 & $2(1.5)$ & $8(6)$ & $8(6)$ \\
\hline $\begin{array}{l}\text { Matemáticas y ciencias } \\
\text { físicas }\end{array}$ & 10 & 7 & $0(0)$ & $0(0)$ & $8(6)$ \\
\hline Música & 8 & 6 & $0(0)$ & $1(1)$ & $6(4.5)$ \\
\hline Química & 2 & 1.5 & $0(0)$ & $0(0)$ & $1(1)$ \\
\hline $\begin{array}{l}\text { Tecnología de récords } \\
\text { médicos }\end{array}$ & 4 & 3 & $0(0)$ & $0(0)$ & $4(3)$ \\
\hline Tecnología médica & 3 & 1 & $0(0)$ & $2(1.5)$ & $1(1)$ \\
\hline Tecnología radiológica & 1 & .75 & $0(0)$ & $0(0)$ & $1(1)$ \\
\hline Total & 133 & 100 & $8(6)$ & $41(31)$ & $73(55)$ \\
\hline
\end{tabular}

* Varios totales incluyen esta categoría

la el uso de la biblioteca. En cambio, educación con una contribución total del $19 \%$, el $3 \%$ estimula mucho el uso de la biblioteca.

Por otra parte, Lenguas y literatura, áreas por naturaleza orientadas hacia un alto uso de la biblioteca, contribuyó al 14\% de los prontuarios, pero de ellos solamente el $1.5 \%$ estimula mucho el uso de los recursos bibliotecarios.

Ciencias sociales y artes liberales, sorpresivamente, tampoco estimulan mayormente el uso de los recursos. Las demás áreas científicas, como matemáticas y ciencias físicas, química y biología presentan una situación semejante.

En la Tabla 4 se presentan los resultados de la encuesta realizada entre una muestra selectiva de la facultad cuyos prontuarios se usaron para el presente estudio. Esta encuesta tuvo por objeto confirmar dos aspectos importantes del estudio: primero, validar la información contenida en los prontuarios sobre modos de instrucción y, segundo, apoyar el supuesto acerca de la poca participación de la facultad en la selección de materiales para la biblioteca.

De 71 cuestionarios que se distribuyeron, sólo se obtuvo respuesta de 27 profeso- res, o sea del $38 \%$ de los encuestados, una cifra bastante baja, pero se decidió utilizarla por considerar que las respuestas obtenidas en los cuestionarios devueltos reflejan el gran medida una tendencia general expresada en los supuestos del estudio.

Además de la respuesta a las dos preguntas del cuestionario (véase el anexo 1), se le pidió al profesor, en la primera pregunta, indicar cuál o cuáles métodos y explicar brevemente si el resultado había sido positivo o no. en la segunda pregunta, si la respuesta era positiva, se le solicitó describir en pocas palabras su participación, y si la respuesta era negativa, expresar las razones por las cuales no participa.

De los 21 profesores que respondieron $\mathrm{Si}$ a la primera pregunta, 9 de ellos, o sea cerca del $43 \%$ aludieron como método adicional el uso de la sala de audiovisuales de la biblioteca para ver películas, videos o materiales semejantes, un método que contribuye al uso de las facilidades físicas de la biblioteca, mas no al uso de los recursos informativos existentes allí. Diez de los profesores expresaron de diversas maneras que motivan el uso de los recursos de la biblioteca, por ejemplo: "Requiero revisar periódicos o revistas,"
Tabla 4

Respuesta a cuestionario para confirmar información en los prontuarios

\begin{tabular}{|l|c|c|}
\hline & Sí & No \\
\hline $\begin{array}{l}\text { 1. ¿Fuera de la metodología que } \\
\text { aparece en el prontuario, utiliza } \\
\text { usted otro(s) método(s), formal } \\
\text { (es) o informal(es), para motivar } \\
\text { el uso de la biblioteca? }\end{array}$ & 21 & 6 \\
\hline $\begin{array}{l}\text { 2. ¿Participa usted activamente } \\
\text { en la selección de los recursos } \\
\text { (libros, revistas, etc.) de la } \\
\text { biblioteca? }\end{array}$ & 18 & 6 \\
\hline
\end{tabular}

"se requieren lecturas o trabajos adicionales," "Los estudiantes deben buscar información específica," "Búsquedas bibliográficas," pero ninguno ofreció una explicación sobre el resultado positivo o no de esa metodología.

A la segunda pregunta sobre la participación de la facultad en el desarrollo de la colección de la biblioteca, 18 profesores contestaron afirmativamente, 6 dijeron que no y 3 no contestaron. La mayoría de los afirmativos comentó que participa recomendando libros dejados por los vendedores, una práctica poco deseable, si el objetivo es tener una colección representativa de lo mejor que hay disponible en el mercado en general.

Varios profesores comentaron que antes participaban recomendando libros y revistas, pero que ahora no lo hacen porque con frecuencia se les dice que no hay presupuesto disponible. Un profesor dio una respuesta muy significativa: "No he sido invitado a participar y desconozco los procedimientos para solicitar adquisición de recursos." Sería importante analizar qué tan generalizada es esta situación en el Recinto.

\section{ANÁLISIS DE LAS REFERENCIAS BIBLIOGRÁFICAS DE LOS PRONTUARIOS}

La Tabla 5 presenta un desglose cronológico de la fecha de publicación o producción de los libros, textos, artículos de revista y videos mencionados en los prontuarios.

Entre los hallazgos más importantes, se destaca el hecho que de los 450 libros que aparecen en los prontuarios, 2 de ellos tienen fechas anteriores de 1940 y un total de 196 , o sea un $43 \%$ son anteriores a 1979. Desde luego, existen categorías de materiales bibliográficos que no envejecen, pues se consideran los clásicos de 
Tabla 5

Cronología de referencias bibliográficas que aparecen en los prontuarios

\begin{tabular}{||l|c|c|c|c|c|c|c|c|c||}
\cline { 2 - 12 } & $\begin{array}{c}1914 / \\
40\end{array}$ & $\begin{array}{c}1945 / \\
59\end{array}$ & $\begin{array}{c}1960 / \\
69\end{array}$ & $\begin{array}{c}1970 / \\
79\end{array}$ & $\begin{array}{c}1980 / \\
84\end{array}$ & $\begin{array}{c}1985 / \\
89\end{array}$ & $\begin{array}{c}1990 \\
/ 93\end{array}$ & $\begin{array}{c}\text { Sin } \\
\text { fecha }\end{array}$ & Total \\
\hline Libros & 2 & 13 & 40 & 141 & 98 & 69 & 18 & 69 & 450 \\
\hline Textos & & & 2 & 5 & 3 & 22 & 15 & 15 & 62 \\
\hline Artículos de revistas & 2 & 1 & 1 & 20 & 15 & 51 & 31 & & 121 \\
\hline Videos & & & & 5 & & & & & 5 \\
\hline Total & 4 & 14 & 43 & 171 & 116 & 142 & 64 & 84 & 637 \\
\hline
\end{tabular}

cada disciplina, pero en muchas áreas del conocimiento actual, no sólo científicas y técnicas, sino un gran porcentaje de libros anteriores a 1990 puede contener información desactualizada. Es interesante notar que 69 libros y 15 textos no aparecen con fecha de publicación en los prontuarios.

Los libros publicados entre 1970 y 1979 son los más utilizados por la facultad, de acuerdo con las referencias encontradas en los prontuarios. Se contabilizaron 141, equivalente a un $31 \%$ en esa categoría de material. Esta situación amerita modificarse para que los estudiantes obtengan información más actualizada.

Un alto porcentaje de artículos de revistas tiene fecha entre 1985 y 1989 , un total de 51 , lo cual representa el $42 \%$. Además, es importante destacar que, acumulativamente un poco más del 32\% de los artículos de revista son anteriores a 1984, un porcentaje bastante alto si se tiene en cuenta la rapidez con la cual se efectúan cambios en las humanidades, las ciencias sociales, la ciencia y la técnica y se reportan en la literatura periódica.

Es importante también recalcar que 52 de los prontuarios analizados indican el uso de libros de texto, una metodología que no contribuye a estimular el uso de los recursos bibliotecarios ni a proceso de investigación (aprender a aprender), tan necesarios e importantes para el individuo en la sociedad contemporánea.

Sorprendentemente, los prontuarios reportan la utilización de sólo 5 videos. Esto podría interpretarse de diferentes maneras, entre ellas que los profesores no usan esta modalidad de enseñanza o que los recursos existentes en la biblioteca no satisfacen las necesidades de los profesores que desean hacer uso de ellos. La investigación, sin embargo, no buscó determinar ese hecho.
En general, debe mencionarse el hecho que cerca del 57\% (349) del total de los recursos bibliográficos reportados por los profesores, son anteriores a 1984, o sea que la información contenida en ellos tiene más de diez años, si se considera que un libro, por lo general, toma unos dos años para publicarse.

Un dato de singular importancia, relacionado con la categoría anterior, es que un poco más del $80 \%(450+62)$ de las referencias bibliográficas reportadas por los profesores se refiere a libros o textos, mientras que sólo cerca del 20 por ciento restante se refiere a artículos de revista y, como ya se dijo, las publicaciones periódicas ofrecen información de mayor actualidad.

\section{CONFRONTACION DE LAS REFERENCIAS BIBLIOGRAFICAS}

A fin de verificar, tanto la disponibilidad en la Biblioteca Juan Cancio Ortiz de los materiales reportados en los prontuarios, como la existencia de ediciones diferentes del mismo material en el sistema bibliotecario de la universidad, y/o ediciones porteriores publicadas pero no disponibles en la biblioteca, se consultaron: el catálogo en línea de la biblioteca y las obras Books in Print y Libros en Venta. Los resultados aparecen en la Tabla 6. De 212 citas bibliográficas seleccionadas aleatoriamente para confrontar en el catálogo de la biblioteca, fue posible identificar un total de 170 , o sea el $80 \%$. Las restantes (42) no se encontraron en el catálogo, lo cual podría interpretarse de varias maneras:

1) El libro es copia personal del profesor y por lo tanto no aparece en el catálogo, aunque sí podrían usarlo los estudiantes por intermedio del servicio de reserva de la biblioteca.
Tabla 6

Confrontación de referencias bibliográficas en el catálogo de la UIPR

\begin{tabular}{|c|c|c|c|}
\hline & & No. & $\%$ \\
\hline \multicolumn{4}{|c|}{ Biblioteca J. C. Ortiz } \\
\hline & Edición igual & 61 & 29 \\
\hline & Edición Anterior & 21 & 10 \\
\hline & Edición Posterior & 16 & 7.5 \\
\hline \multicolumn{4}{|c|}{ En otra biblioteca UIA } \\
\hline & Edición igual & 34 & 16 \\
\hline & Edición Anterior & 16 & 7.5 \\
\hline & Edición Posterior & 22 & 10 \\
\hline \multicolumn{2}{|c|}{ No en el sistema UIA } & 42 & 20 \\
\hline \multicolumn{2}{|c|}{ Total } & 212 & 100 \\
\hline
\end{tabular}

2) La obra aparece en la bibliografía, pero quizás el libro ya no exista en la biblioteca porque se extravió.

3) Las referencias del prontuario fueron tomadas de un prontuario hecho por otro profesor que ya no está en la universidad y los libros citados eran propiedad del profesor anterior.

4) La referencia se tomó de una fuente distinta a la biblioteca y nunca se confirmó la disponibilidad de la obra localmente. La presente investigación no pensó en profundizar en esta importante área y sería deseable indagar esa situación en un trabajo posterior.

Otro aspecto que se destaca en la tabla es la variación en cuanto a las ediciones de los materiales bibliográficos reportados en los prontuarios.

Solamente en el $29 \%$ de los casos hay correspondencia entre lo reportado y lo existente en la biblioteca. Del $10 \%$ de las referencias reportadas hay ediciones posteriores a las existentes en la biblioteca, lo cual es significativo en cuanto a la actualidad de la colección y la posible participación de la facultad en el desarrollo de la misma. Por fortuna, de las referencias reportadas el $7.5 \%$ está representado por ediciones posteriores en la biblioteca, un hecho relativamente común en las bibliotecas académicas y que sirve para señalar posibles deficiencias en el sistema de reportar nuevas adquisiciones a los profesores y/o la renuncia de algunos miembros de la facultad a usar nuevas ediciones de libros, a veces porque éstas contienen material nuevo que 
puede alterar un conjunto de rutinas establecidas. El autor de la presente investigación ha tenido esta experiencia en otras instituciones.

Un hallazgo importante fue que de las 170 referencias verificadas, 68 , o sea el $40 \%$, no se encuentran en la biblioteca Juan Cancio Ortiz, aunque sí aparecen como existentes en otros Recintos de la Universidad Interamericana. No se verificó el uso del préstamo interbibliotecario para obtener estos materiales porque la investigación no estaba dirigida al análisis de este aspecto. La no disponibilidad de dichos materiales en San Germán sí es un hecho muy significativo que deberá investigarse para poder determinar sus orígenes y posibles efectos negativos en el desarrollo del proceso enseñanza-aprendizaje.

\section{VERIFICACION DE REFERENCIAS EN OBRAS DE CONSULTA EN ESPAÑOL E INGLES}

A fin de establecer con mayor certeza la existencia en el mercado de ediciones más recientes de una muestra (212) aleatoria representativa de las obras citadas en los prontuarios se consultaron las obras Books in Print y Libros en Venta para libros en inglés y español, respectivamente. La Tabla 7 ilustra los hallazgos más significativos.

Al igual que en la Tabla 6, en esta Tabla puede verse que las obras de referencia mencionadas incluyen ediciones más recientes de las que aparecen en los prontuarios. En el caso de los libros en español, de un total de 58 verificados, 11 , o sea el 5\% aparecen en Libros en Venta como ediciones más recientes. De los libros en inglés, 25 , o sea el $12 \%$ aparece en Books in Print como ediciones más recientes. Significativamente, 92 de los libros verificados, o sea un poco más del $43 \%$, no aparecen en la edición más reciente que tiene la Biblioteca de Libros en Venta y Books in Print, lo cual casi siempre indica que el libro está agotado, no se publica más y difícilmente puede conseguirse por los canales comerciales regulares. Esta sería, entonces, otra área que requiere atención para establecer el alcance y el tipo de problema que la situación presenta.

Tabla 7

Verificación de referencias en obras de consulta

\begin{tabular}{|c|c|c|c|c|}
\hline Fuente & Incluida & Edición & Total & Porcentaje \\
\hline \multirow{3}{*}{$\begin{array}{l}\text { Libros en } \\
\text { Venta } 1990\end{array}$} & Sí & Igual & 26 & 12 \\
\hline & Sí & Posterior & 11 & 5 \\
\hline & No & & 21 & 10 \\
\hline \multicolumn{3}{|l|}{ Sub-total } & 58 & 27 \\
\hline \multirow{3}{*}{$\begin{array}{l}\text { Books in } \\
\text { Print 1990-91 }\end{array}$} & Sí & Igual & 58 & 27 \\
\hline & Sí & Posterior & 25 & 12 \\
\hline & No & & 55 & 26 \\
\hline \multicolumn{3}{|l|}{ Sub-total } & 138 & 65 \\
\hline $\begin{array}{l}\text { Books in Print } \\
\text { anteriores }\end{array}$ & & & 16 & 8 \\
\hline \multicolumn{3}{|l|}{ TOTAL } & 212 & 100 \\
\hline
\end{tabular}

\section{CONCLUSIONES}

Este estudio demostró que los prontuarios contienen información muy valiosa sobre diferentes aspectos de la actividad académica en la universidad y que los diferentes análisis presentados pueden servir de base para investigaciones adicionales y la introducción de algunas reformas en los modos de presentación de los contenidos de los cursos, así como en la interrelación que debe existir entre la facultad y los bibliotecarios para el mejor desarrollo del proceso enseñanza-aprendizaje.

La investigación reveló, de varias maneras, que los prontuarios, en términos generales, no estimulan el uso de la biblioteca, que se usa el libro de texto en un alto porcentaje de los cursos que se ofrecen en el Recinto y que parece haber poca participación de la facultad en el desarrollo de la colección de la biblioteca. Resultados que responden a la pregunta de investigación formulada inicialmente y confirman los supuestos básicos que dieron sustento a este trabajo.

El estudio puso en evidencia, además, que las metodologías más usadas por la facultad del Recinto como: libros de texto, lecturas asignadas, laboratorios $y$ ejercicios no contribuyen al uso de la biblioteca y que muchos de los libros disponibles para los usuarios de la biblioteca pueden contener información muy desactualizada.

Es importante, también, destacar el hallazgo reportado en relación con el mayor o menor estímulo que los diferentes departamentos y programas parecen darle al uso de los recursos bibliotecarios.
Haría falta no sólo experimentar con otras metodologías, sino buscar los mecanismos adecuados para lograr una mayor interacción entre los estudiantes, los bibliotecarios y la facultad. en ocasiones, la falta de uso de la biblioteca puede deberse al desconocimiento que la facultad y los estudiantes tienen de los recursos existentes y cómo utilizarlos, y los bibliotecarios de la situación que se presenta a diario en el salón de clase.

\section{RECOMENDACIONES}

Las principales recomendaciones surgidas a raíz del presente estudio son las siguientes:

1. Estimular la evaluación de las metodologías utilizadas actualmente por la facultad, a fin de introducir cambios que generen un mayor uso de la biblioteca. En este sentido, es muy importante recalcar la imperiosa necesidad que todos los estudiantes que en el futuro se gradúen de la universidad, demuestren proficiencia en el acceso, evaluación y uso de diferentes tipos de información.

Este concepto es congruente con la tendencia actual, generalizada en el mundo académico y activamente estimulada por Middle States, hacia la alfabetización e información (Information Literacy).

2. Desarrollar estrategias que estimulen la mayor utilización de la biblioteca, no sólo por medio del prontuario, sino a través de un amplio y relevante programa de instrucción en el uso de la biblioteca, la información y los libros, preferiblemente con la participación activa de los profesores, quienes co- 
nocen a fondo sus respectivas especialidades y fuentes de información, así como las necesidades de los estudiantes.

En relación con este asunto, se recomienda, además, estudiar la posibilidad de establecer un curso con crédito para estudiantes de nuevo ingreso, sobre el uso de bibliotecas, libros e información.

3. Procurar una mayor participación de la facultad y coordinación con los bibliotecarios, en el desarrollo de la colección de materiales bibliográficos y no bibliográficos indispensables para el normal desarrollo del proceso enseñanza-aprendizaje.

4. Apoyar diferentes iniciativas y estrategias tendientes a actualizar la colección y los servicios de acceso a la información, de tal manera que la comunidad académica disponga siempre de lo más actualizado.

5. Nombrar un comité asesor de la biblioteca que se encargue de establecer una mayor interacción entre los recursos educativos, la facultad, los estudiantes y la administración y apoye el desarrollo integrado de los recursos informativos, su acceso, evaluación y uso.

6. Estudiar la posibilidad de incrementar el uso de metodologías de enseñanza como el trabajo escrito, los proyectos, el estudio de casos, los estudios individuales, la búsqueda en biblioteca y las asignaciones en revistas, que contribuyen mayormente al uso de la biblioteca.

Igualmente, como la computadora no se reporta como medio de instrucción, se recomienda estudiar las causas de esta situación y buscar alternativas para darle impulso a este importante medio.

7. Estimular el cumplimiento de las directrices sobre la preparación de los prontuarios y poner énfasis en la necesidad de incluir una metodología y referencias bibliográficas y no bibliográficas recientes, sobre todo a revistas y materiales que contienen información de mayor actualidad.

8. Finalmente, se recomienda estudiar la necesidad y conveniencia de adoptar en el Recinto, un formato de presentación de trabajos de investigación por parte de los estudiantes. Esto no sólo les capacita para algo muy útil en la vida profesional, sino que le imprime uniformidad a la presentación de trabajos en el Recinto.

\section{REFERENCIAS}

"Determinig sample size for research activities". En Educational and psychological measurement, 30 (1970): 608-610.

Golden, Barbara. "A method for quantitatively evaluating a university library collection”. En Lib. Res. Tech. Serv., 18 (Summer 1974): 268-74.

Lauer, J. D., Merz, L. H., and Craig, S. L. "What syllabi reveal about library use: a comparative look at two private academic institutions." En Research Strategies, 7.4 (1989): 167-174.

Rambler, Linda K. "Syllabus study: Key to a responsive academic Library.” En J. Academic Librarianship, 8.3 (1982): 155-159.

Sayles, Jeremy W. "Course information analysis: foundation for creative library support.” En J. Academic Librarianship, 10.6 (1985): 343-345.

Spencer, Robert C. "The teaching library." En Lib. Journal, 103 (May 15, 1978): 1022 .

Whaley, John H. jr. “An approach to collection analysis.” En Lib. Res. Rech. Serv., 25 (July/Sept. 1981): 330-38.

\section{BIBLIOGRAFÍA ADICIONAL}

Nota: Las siguientes referencias presentan diferentes aspectos relacionados con el tema de la investigación y tienen como objeto orientar sobre cambios posibles en metodologías del proceso educativo.

American Business Communication Association. How to teach small group decision-making in a basic business communication class. Urbana: ABCA, 1981.

Evans, John E. Information resources, perspectives and strategies for graduate educational research: a course proposal outline and rationale. Memphis, Ten.: State University, 1986.

Fortune, Gwendoline. Performance contract or syllabus? A comparison of student achievent in social science courses. Ed. D. Practicum, Nova University, 1977.

Fox, Lynne and others. "Partnership for the future: information literacy in nursing education." Paper presented at the Annual Conference of the Northern Rocky Mountain Educational Research Association. (Greely, Col.: Oct. 1990).

Library research curriculum materials for a one credit course. compiled by Jacqueline Gabryck and others. Albany: State University of New York Libraries, 1981. 
Lory, Alice and others. "We must all teach writing." Paper presented at the Annual meeting of the Conference on College composition and communication (28th, Kansas City, Missouri, March 31-April 2, 1977).

Lowry, Anita K. "Beyond BI: information literacy in the electronic age.” En Research Strategies, 8.1 (Winter 1990): 22-27.

Melhado, L. Lee. "Chemical composition: improving the writing and literature searching skills of studentes in the chemical sciences." En Journal of chemical education, 57.2 (Feb. 1980): 127-28.

Miko, Chris J. "Introducing end-user searching through course syllabi." Paper presented at the Annual meeting of the Michigan Academy of Science, Arts, and Letters (90th, Mount Pleasant, Mich. March 14-15, 1986).

Laughlin, Jody. "The use of current media in literature and composition at the community college level." Paper presented at the Annual meeting of the popular culture association. (18th, New Orleans, Lous., March 23-26, 1988).

Niles, Nancy and Trudy E. Jacobson. "Teaching critical thinking in libraries.” En Research Strategies, 9.4 (Fall 1991): 198-201.

Palmer, Richard. Integrating library acquisitions with the curriculum. 1976.

Waggener, Joe and others. Media and technology for teaching. Course manual for EDM/EDT 343/443/543. Oxford, Ohio : Miami University, 1992.

Wilson, Lizabeth. Teaching librarians to teach: a course in library use introduction. Champaign, Ill. : University of Illinois, Graduate School of Library and Information Science, 1991.

\section{Recomendaciones para mejorar el uso de los recursos educativos}

Además de las recomendaciones surgidas como resultad de la investigación, se ofrecen enseguida varias sugerencias para motivar el uso de los recursos educativos del Recinto.

En primer lugar, y a manera de marco filosófico que abarca el concepto de uso de la biblioteca, debe puntualizarse la importancia que actualmente se le da en diferentes lugares del mundo a la habilidad que todo ser humano debe tener para accesar, evaluar y usar información.

Con la avalancha de información que a diario se genera en el mundo y el impacto que ella produce en todos los sectores de la actividad social, política, económica, científica y tecnológica, la supervivencia individual en el competitivo entorno de la actividad laboral contemporánea, depende en gran medida de la habilidad de cada uno de nosotros para adaptarse a los cambios constantes. Y sólo quienes posean destrezas para mantenerse al día por medio de la información podrán competir con éxito.

La universidad debe, por lo tanto, lograr articular un conjunto de estrategias que le permitan a sus egresados obtener esas destrezas como parte integral de su for- mación académica. Desde el primer año hasta los estudios de posgrado, cada estudiante debe exponerse a diferentes modalidades de acceso a la información manual y computarizada que le permita, a la vez, desarrollar su pensamiento crítico (evaluación de la información), la habilidad de investigar (aprender a aprender) y usar esa información para producir nueva información y tomar decisiones.

Desde luego, la participación de la facultad en el proceso es crucial. Sin su aporte motivador mediante las clases y el ejemplo, es difícil que el estudiante comprenda la importancia de obtener las destrezas requeridas. De ahí que también se recomiende lograr que la facultad sea activamente consciente de esta importante responsabilidad.

También, por parte de todas las personas que directa o indirectamente están implicadas en el servicio a los usuarios de la biblioteca, debe existir el compromiso intelectual y la firme determinación de no ahorrar esfuerzos para que puede cumplirse con la mayor eficacia posible su misión académica.

Uno de los mejores medios para lograr el aprendizaje de las destrezas necesarias, es por medio de un programa modelo de instrucción en el uso de la biblioteca, los libros y la información. Este programa debe contar con el apoyo y la participación activa de la facultad.

Las directivas del Recinto deben, igualmente, dar su respaldo intelectual y financiero para que un programa de esta naturaleza alcance sus objetivos.

Fuera de estas recomendaciones, el plan de estímulo al uso de los recursos debe incluir un análisis profundo acerca de las necesidades reales de los usuarios de la biblioteca en cuanto a facilidades físicas, de materiales impresos y no impresos para sus clases e investigaciones y el uso de las nuevas tecnologías para el acceso y manejo de la información.

Finalmente, uno de los mejores motivadores del uso de la biblioteca lo constituye, sin lugar a dudas, el buen servicio que se preste a los usuarios. Se recomienda, entonces, evaluar a fondo la calidad y cantidad de los servicios que recibe la comunidad universitaria e introducir las modificaciones requeridas para poder satisfacer las necesidades de todos los usuarios. 